\title{
Perceived use of health care service: barriers to access prehospital care in Jimma City, Oromia Region of Ethiopia
}

Shemsedin Amme Ibro ( $\square$ shamsuamme@gmail.com )

Jimma University

Lippi Matthew

University of Colorado Anschutz Medical Campus

Sheka Shemsi Shemsi

Jimma University

Adugna Olani Akuma

Jimma University

Tura Koshe Haso

Jimma University

Abdulwahid Awol Ahmed

Jimma University

Esmael Ahmed Simbiro

Jimma University

Tolasa Takele Moga

Jimma University

Muktar Beshir Ahmed

Australian Centre for Precision Health, University of South Australia, Adelaide

Habtamu Jarso Hebo

Madawalabu University

Sultan Suleman Waga

Jimma University

Gemechis Melkamu

Jimma University

Ebrahim Yimam Reta

Jimma University

Shimelis Legesse Chala

Jimma University

Nee-Kofi Mould-Millman

University of Colorado Anschutz Medical Campus 


\section{Research Article}

Keywords: Prehospital care, Out-of-hospital-emergency-care, Access Barriers, Ambulance service, Emergency Medical Service, Ethiopia

Posted Date: January 31st, 2022

DOI: https://doi.org/10.21203/rs.3.rs-897121/v2

License: (c) (i) This work is licensed under a Creative Commons Attribution 4.0 International License.

Read Full License 


\section{Abstract}

Background: African nations experience a significant proportion of the global burden of death and disability. The provision of prehospital emergency care has been shown to partially reduce excess morbidity and mortality. However, access to prehospital care in Africa is still limited. This study sought to identify barriers to access prehospital care in the city of Jimma, Ethiopia.

Methods: This is an interview-based qualitative study of key prehospital stakeholders in Jimma, conducted in February 2018. A purposive sample of individuals from the community and local ambulance organizations was selected for interviews. Interviews were conducted in local languages, translated into English, and then coded for consistent themes.

Results: All respondents felt that prehospital care was difficult to access and therefore infrequently utilized. This was due to a combination of a limited number of ambulances, the lack of a toll-free emergency number, the lack of a single organized EMS system, inconsistent and limited training of ambulance crews, public mistrust of the existing system, poor road infrastructure, and limited public understanding of the role of prehospital care. Respondents suggested that establishment of a formalized prehospital care system, investment in infrastructure, establishment of a toll-free emergency number, public awareness campaigns, and more widely available emergency medical training, which were feasible solutions to these current barriers to access.

Conclusion: Multiple barriers to accessing prehospital care were identified in Jimma. Establishing a formalized, well-resourced prehospital system in parallel with improving community capacity and knowledge building were suggested solutions to improve access. Hence, interventions to improve prehospital emergency care delivery should ideally target these identified barriers and proposed solutions.

\section{Background}

African nations experience a disproportionately large share of the global burden of death and disability [1]. The high burden of time-sensitive injury and illness in low-income countries has been cited as a compelling reason to implement emergency medical care systems [2]. In many African countries, higher proportions of deaths occur before arrival to a health facility, when compared to low and middle-income nations of other continents [3-5]. While the cause is multifactorial, under-developed prehospital care systems in Africa is a likely contributor to the disproportionate burden of morbidity and mortality $[2,6,7]$.

Prehospital care plays an essential role in emergency medical care by providing on-scene stabilization and safe, rapid transportation by trained ambulance crews to an appropriate health facility [6-8]. Therefore, many African and international expert groups have advocated for the creation and integration of prehospital care into existing healthcare systems $[6,7,9]$. Yet, prehospital care remains uncommon in most African countries, with less than $9.0 \%$ of the population having reliable access to prehospital care [8]. Several barriers to accessing prehospital care have been reported across Africa [7, 9-12], most 
commonly including poor public communication infrastructure, limited public education, and inadequate prehospital response units [6-8, 10-14].

Ideally, the development of a robust prehospital care system should be guided by evidence; however, the development of emergency care in Africa has been inadequately studied [6, 15-17]. Evaluation of existing systems and services, as well as the community's experience with care, are key steps towards the formation of a robust prehospital care system [8]. Specifically, understanding the barriers to service utilization is an important initial step $[6,12]$. This study assessed the community's perceptions of access to prehospital care services in Jimma, Ethiopia.

\section{Methods}

\section{Study settings, design and population}

A qualitative study was conducted to assess perceptions about prehospital emergency care access in Jimma City between February 5 and 23, 2018. Key stakeholders for prehospital emergency care, including organizations offering ambulance service, community members and healthcare workers were involved in the study. Eligible informants were 18 years or older, who resided or worked in Jimma for at least one year, and spoke Afan Oromo or Amharic languages, and there were no exclusion criteria unless the participants refused to participate.

A purposive sampling technique was used to select 68 key informants from a variety of relevant settings in Jimma, including the community, police department, public schools, public healthcare facilities and organizations providing ambulance services in the city. Community (kebele) leaders assisted in recruiting appropriate participants within the 12 administrative kebeles of Jimma. In general, study participants were: community members and leaders; community health extension workers; school principals and teachers from primary and secondary public schools; traffic police and community police officers from two police departments; academicians who were originally healthcare professionals from a local university; healthcare professionals working at public healthcare facilities, including public primary health centers and hospitals; and representatives from ambulance services including administrative officials, coordinators and ambulance drivers from two public and three facility-based ambulance services.

\section{Data collection}

Two semi-structured interview guides were developed - one for individual in-depth interviews, and another for focus-group discussions. General topics participants were asked about awareness of prehospital care, personal experience with prehospital care, perceived availability and accessibility, barriers to access, and suggestions to overcome barriers. We used focus group method to get information from different group respondents' perspectives that can contribute to a better understanding of issues under investigation, while individual interviews used to illicit information related with personal experiences and to get replies to questions which are too officials. 
The interview guides were first prepared in English, then translated to Afan Oromo and Amharic, further tailored to fit the background or organizational context of the relevant informant, and pretested for clarity and flow. The tools were forward and backward translated by different individuals to ensure consistency between the original and translated versions.

Focus group discussions (FGD) were conducted with small groups consisting of 5-6 community members. Three 'closed door' FGDs were conducted at a hall in the community. Each FGD discussion lasted for 45-60 minutes, and was audio-recorded after verbal permission was obtained from the participants and supplemented with facilitator note-taking. FGDs were conducted by eight trained facilitators who were healthcare professionals (one moderator and note-taker for each session), and who were fluent in both local Afan Oromo and Amharic languages.

In-depth individual interviews were conducted with officials from ambulance service organizations, healthcare professionals, and content experts. In-depth interview sessions were taped after oral consent was obtained from the interviewee and notes were taken. Each session lasted for 30-45 minutes and was conducted by the lead investigator. Interviews and FGDs were conducted until thematic saturation was reached.

\section{Data analysis}

Audio records and interviewer notes were translated and transcribed into English using the Microsoft Office Word process (Microsoft Office 2016). Then transcripts were back-translated to ensure consistency between the original and transcribed translations[12]. Before actual data analysis, the lead author fully explored the transcribed data, and identified key themes that were used to construct the coding frameworks using a content analysis approach.

The lead author started coding data and framing it into identified key themes. Then, the lead investigator provided a detailed meaning of each theme and code to a second investigator who would also be coding the data. Next, a second researcher started coding the data using the predefined codes and themes on the same transcripts that were previously coded by the lead author. Then, a third independent researcher assessed consistency between the two coding researchers and this continued until consensus was reached. Finally, the lead author explored and integrated the data into the final major themes and subthemes. An inductive analysis was used to aggregate data into eleven sub-themes that were organized into three larger themes including awareness and personal experience of participants with prehospital care, barriers to access, and perceived solutions to overcome the barriers. Thematic saturation was determined when coding researchers reached consensus that the interview data failed to identify new opinions from participants. Finally, a report on the findings was written by the lead investigator and distributed to the rest of the study team.

Informed verbal consent was obtained from the participants, which was approved by the Jimma University, Institute of Health IRB. All methods were carried out in accordance with relevant guidelines and 
regulations.

\section{Results}

Overall, there were 52 individual interviews, and 3 focus group discussions completed representing 68 total participants. Out of them, 45 were males and 36 individuals were from the community. Their occupations (roles) were Community leaders, Members of community (merchants, teachers and police officers), Officials and Healthcare workers; Table 1 presents the community roles/occupations of the study participants. Findings are presented below and organized into three main thematic areas, each with sub-themes.

Table 1: Characteristics of the study participants

\begin{tabular}{|lc|}
\hline Characteristics of the participants & Number \\
Sex & \\
Male & 45 \\
Female & 23 \\
Total & 68 \\
Roles (Occupation) & \\
Members of the community (merchants and other self-employed) & 14 \\
Community leaders & 12 \\
Police officers & 6 \\
School teachers & 4 \\
Officials from organizations & 10 \\
Health professionals & 10 \\
Community health extension workers & 8 \\
Ambulance drivers & 4 \\
Total & 68 \\
\hline
\end{tabular}

\section{Awareness and personal experience with prehospital care}

\subsection{Prehospital care awareness}

Respondents were asked about prehospital care and its role in saving lives. Most respondents knew about the concept of first-aid being provided at a scene, while health professionals often added prehospital transportation and care. Respondents agreed that it was important to give care in the prehospital setting to preserve life and prevent complications; they stated that helping someone in an emergency is a moral obligation. 


\subsection{Personal utilization experience}

Respondents were asked about personal experiences with prehospital services, with regard to ambulance service. Most of the respondents neither utilized the service nor knew the phone number to call for an ambulance at the time of their interview. However, a few respondents did have experience of using an ambulance services. Of those, most had used their personal phone to call for an ambulance.

"I had experience (of using an ambulance), while I was in labor [...]. Often, I also call for others, but it is less available during night time." (A community administration officer)

\section{Barriers to access}

Several barriers to accessing prehospital care were often identified by respondents. These include limited public awareness, infrastructure and organizational issues, and a lack of trained providers.

\subsection{Public information and perceptions}

Respondents felt that there was uncertainty in the community related to when and how to initiate a call to the EMS system and what type of care to expect in the prehospital settings. Interviewed officials knew that there were toll-based landline phone numbers to reach ambulance services. However, only a few respondents knew of those phone numbers and none of them could recall those numbers having been publicly advertised. Overall, they felt that the ambulance operator's phone number was not commonly known and this was true even among healthcare professionals. In spite of this, ambulance drivers reported frequent requests for an ambulance through phone calls; although they also reported prank calls. Furthermore, respondents identified misconceptions in the community with regard to contacting ambulance services. Some believed that only members of the Red Cross Society (RCS) and those who personally knew healthcare professionals could use or have access to an ambulance. Others felt that ambulances were only meant for certain emergency conditions; maternal and pediatric emergencies being the most frequently cited. Difficulty in contacting services due to lack of a toll-free universal emergency number is the most commonly raised system-related barrier. Unfortunately, interviewed health professionals felt that building public awareness of how to contact and the purpose of an ambulance system would be difficult in the absence of a formalized prehospital Emergency Medical Service (EMS) system.

"Majority (community members) do not have an ambulance phone number [...], but it should have to be advertised publicly, through mass-medias, at different community events like meeting stages and 'Idir [Idir 
is a form of indigenous community organization that gathers or connects people in times of grief and joy]". (One of the community leaders)

\subsection{Inadequate system organization and infrastructure}

\subsubsection{Inadequate system organization}

Poorly organized prehospital care system resources (in terms of personnel, logistics, communication, and transportation) and poor integration with facility-based emergency care was another major barrier to access. Respondents felt there was no uniform structure for prehospital care, upon which the many providers and resources could be organized. They commonly mentioned ambulance scarcity relative to the needs in the community. Furthermore, there was often no back-up ambulance, should one need to be taken out of service for maintenance or repairable damage. Additionally, the available ambulances lacked essential emergency supplies and medications, and were not always staffed with a trained crew. Officials also mentioned limited budget allocation to ambulance services, which did not afford operational expenses for ambulances such as fuel and maintenance costs. For instance, an officer mentioned that, at the time of this interview each publicly operating ambulance receives about 50,000 Ethiopian Birr for one fiscal year; perhaps this is less than $1200 \$$.

"[...] there is only one ambulance, this cannot address needs in the community (...) most of the time this itself becomes out of service; in such an event, and we don't have another option." (A community leader)

Respondents mentioned that hospital-based ambulances lacked formally allocated phone numbers, whereas, public ambulances use regular landline phone numbers. However, they mentioned that those phone numbers are often not readily accessible for calls. Respondents revealed that phone operators either do not answer calls or challenge the callers as if he/she mistakenly called over a wrong number, particularly during a night-time when optional commercial transportation is not available. Nevertheless, regular phone numbers are not free of airtime charges and are not easily memorable in emergency situations, as interviewed professionals have stated.

"We call them so many times, sometimes they refuse to come... but often they do not answer the calls." (One of the community leaders)

\subsubsection{No standard regulation and protocol}

According to officials and professionals, there are no statewide or local regulatory standards enforcing minimum standards of staffing and logistics for prehospital care. Apart from RCS owned ambulances, which were equipped with first-aid kits and trained volunteer first-aiders, there was felt to be a great deal of variability between crews and emergency supplies. Opinions from respondents revealed that a lack of protocols meant that care may be provided inconsistently or incorrectly, and patients' access to safe and 
professional care cannot be ensured. In addition, there is no legislation that protects prehospital care providers.

"There is no legislation for ambulance service. I am not aware of the existence of regional or national legislation." (A Medical Doctor)

\subsubsection{Delayed response to the scene}

Another access barrier identified was delayed response times. According to community members, it could sometimes take up to 2 hours for an ambulance to arrive, and occasionally it would not arrive at all. Drivers and officials attributed this to a high demand for ambulance services but a limited supply of ambulances. These long response times were a major cause for community dissatisfaction and preference for commercial transportation over the ambulance, according to the respondents.

"[...] usually, they did not come at all, even if they did, they would arrive after labor was already complicated." (A midwife at a public health center)

\subsubsection{Poor roads and transportation options}

Commercial transportation such as taxis are the most commonly used as most of the respondents mentioned. Respondents mentioned difficult roads as another access barrier, compelling community members to prefer commercial taxis over ambulances despite the difficulty of affordability. Often drivers find it hard to navigate to reach the community due to absent or difficult roads within the township.

"There is high interest to use the ambulance in the community, but inner roads between blocks in the city are not suitable to allow ambulances. [...] Often communities are forced to convey laboring women using homemade couches [...] I remember there was a woman who gave birth on the way to the health center, while being carried on the homemade couch." (Health professional at Public health center)

\subsection{Provider related barriers}

\subsubsection{No trained prehospital providers}

Healthcare workers' practices were also among the commonly reported barriers to access. Respondents felt that only RCS ambulances were routinely staffed with individuals who had been trained in first aid and could provide prehospital care. However, several respondents felt that the first aid training of RCS crews was insufficient to ensure safe and proper care during emergencies. According to respondents, the lack of trained ambulance crew was due to limited opportunities to train paramedics and emergency medical technicians (EMTs) locally. Hence, patients did not receive professionally provided initial and ongoing care either due to lack of providers or supplies in the ambulance, as mentioned by respondents. A few respondents also felt that the drivers also lacked proper ethical training and felt that most of the drivers were negligent, lacked compassion and commitment, and were disrespectful to clients. Police officers in particular stated that ambulance drivers were often cited for inappropriate use of sirens, driving 
recklessly, and hauling non-medical items such as illegal drugs and alcohol in ambulances. However, interviewed ambulance drivers and other respondents did not agree with these allegations.

"I called him (the driver) as my sister was in labor; he responded that the ambulance was out of service due to maintenance, so we were compelled to use a commercial taxi. But, afterwards, I saw him while I was on the way to the hospital. He was transporting an administrative person in that same ambulance." (A community health extension worker)

Ambulance misuse is another commonly referred provider-related access barrier to prehospital service. Respondents mentioned ambulances are often misused for non-medical purposes. Most of them either witnessed or were aware of why, how, and by whom ambulances are misused. According to them, ambulances are commonly misused by administrative officials as regular service cars to haul medical and non-medical supplies for official purposes or domestic consumption. Some of them mentioned that ambulances are occasionally involved in transporting security personnel during civil unrest. Interviewed officers have confessed to the existence of the practice, often not on the behalf of their good wishes, but compulsions from administrative officials. They felt lack of: awareness, strong legislation, and administrative commitment to halt and, scarcity of optional transportation as major reasons for ambulance misuse.

"Using ambulances as office service has now become a habitual practice [...]." (A physician at the public hospital)

\section{Perceived solutions to overcome the barriers}

A wide range of solutions were suggested by respondents to improve access to prehospital care. This includes improved: system arrangement and resource allocation, human resource capacity, legislation, and public education.

"Much has to be done to make the ambulance service accessible to the community." (A Nurse)

\subsection{Improved coordination and system arrangement}

Respondents called for improved resource organization and coordination for a better prehospital system and improved access. They felt that having multiple ambulance providers with varying levels of resources was inefficient. They suggested integrating these organizations under a common public access point and a single leading agency to improve coordination of these overstrained providers. They called for the establishment of a call center operated on a toll-free emergency number so that access would be improved. They felt that this was best done through new legislation that brought all providers onto a common platform and working system. 
"I think all those independently operating service providers have to be integrated under one system. [...], but system integration is possible. This requires creating a common working platform; there should be legal enforcement, guidelines and politics and the provider's commitment." (An official from ambulance organization)

\subsection{Improved resource allocation and infrastructure}

Improving resource allocation to prehospital care was another common solution. This included increasing the number of ambulances, care providers, and emergency resources. Officials also suggested increasing budget allocations to ambulance services, while community members felt investing in basic infrastructure such as proper road constructions would do more to improve access to emergency transportation.

"Everybody wants to use the ambulance during emergencies, due to the scarcity of ambulances. [...] the infrastructure, especially, roads inside the city are not suitable and not adequate. There is no universal emergency number. I think all these should be improved; the government has to invest here." (A community member)

\subsection{Improved healthcare providers}

Respondents felt improving EMS personnel would require investment in pre-service or in-service training to increase the quality and uniformity of prehospital emergency care. Although few respondents felt integration of prehospital care courses into existing pre-service training curriculum would improve the availability of professional providers. On the other hand, most of them feel that continuous on-service training would improve the quality of providers.

"I don't know why such training (paramedics training) is still lacking in our country. [Don't] Policymakers and planners believe in the necessity of prehospital care services to our community? [...]. I think this is a time to move forward." (A health professional)

\subsection{Improved regulations}

Respondents suggested standards for personnel, equipment, practice, and training to regulate prehospital care either at the state or local level. Interviewed officials and professionals also suggested prehospital care protocols to standardize care. Others also recommended legislation and administrative commitments to protect EMS resources from misuse.

"[...] and also, there should be guidelines for ambulance service and care at our facility level too. [...] One day we will end this practice (ambulance misuse), but this may take time, and it needs strict regulations and commitment." (A Nurse coordinating an ambulance) 


\subsection{Public education and capacity building}

Most of the respondents supported public awareness campaigns to create a basic understanding of emergency conditions and when and how to access care. They also recommended community capacity building through first-aid training, so that community members can begin to initiate care even before an ambulance arrives.

"The public at large does not have awareness. I have not seen any attempt to (to create public awareness) [...] no interventions to engage the community [...], all these needs to be improved, the public at large should be aware of basic lifesaving skills, a first aid training program for the community." (A Police officer)

\subsection{Improved evidence generation}

Interviewed professionals felt it was critical to collect and synthesize information to better understand the burden of disease and the status of existing services and systems. They believed that this would allow for the identification of gaps in the current system and lead to essential policy changes to strengthen the prehospital system.

"There should be improvements, but it have to be evidence based. Still, further research and investigations have to be done to establish bases and better solutions." (A Health professional)

\section{Discussion}

Access to timely emergency care can be influenced by the perception of, and the ease of access to prehospital care during emergencies [12]. The present study assessed the awareness and perceived barriers to access and community-generated solutions to improve access to prehospital care services in Jimma, Ethiopia.

We identified that prehospital care is under developed and still a new concept in Jimma city, Ethiopia. There is lack of awareness regarding the role of prehospital care and initial emergency care at scene in general population in Jimma city. However, this is one of the major factors that determine seeking and accessing emergency care at the community level[12], as also reported by previous studies in Addis Ababa, Ethiopia[18] and in other African countries[10-12]. To improve public awareness, respondents of this study suggested public education and community-based first aid training programs, which have been also recommended to improve access to prehospital care across Sub-Saharan Africa [6, 7].

It has been known that there is a dire need for prehospital care in Sub-Saharan Africa, and this will continue to mount because of the epidemiological transition from communicable diseases to timesensitive non-communicable diseases and injuries[6]. As a result of this, countries like Ethiopia should invest in proper EMS system development. Our study identified a fragmented system that lacked a common emergency number linked to a single responsible EMS agency, high demand-low availability of 
ambulances, and lack of trained care providers as major barriers to access prehospital care in Jimma city. Having a functional universal toll-free emergency number is critical to ensure that citizens and healthcare personnel can reach EMS resources for medically-appropriate emergency transportation [19]. Hence, in the present study area, to establish an effective prehospital care system, one well-publicized, toll-free emergency number should be made publicly available along with the respective responsible EMS agency. In resource-limited settings of African nations such as Jimma City, establishing a region-specific and context-appropriate emergency medical dispatch (EMD) system has been advocated as a feasible solution to implement effective prehospital care [20]. Fortunately, previously established ambulance dispatch system by the fire department [21], and private providers [22] in Addis Ababa, Ethiopia has proved the feasibility of a local EMS system in Jimma as well. Moreover, experience from developing nations shows locally available resources can be tailored to build effective EMS systems, as in Punjab region of Pakistan [23], and in Jaffna, Sri Lanka[24]. However, there should be efforts on region-specific evidence generation, on the burden of emergencies and the demand for care, and advocacy to create awareness and commitment among stakeholders $[6,7,12]$, to realize this in Jimma city.

The fact is that, if effectively implemented in resource constrained settings; prehospital care system can augment the emergency care system and improves patient outcomes $[25,26]$. However, lack of medical personnel and equipment will reduces the lack of medical personnel and equipment reduces the efficiency and effectiveness[6], and can affects patient's access to safe and appropriate professional care. As of other Sub-Saharan African nations [6, 8, 27, 28], we identified scarcity of ambulances, lack of trained professional provider and medical equipment, for which community enforced to use commercial transportation such as taxi over ambulances as a means of emergency transportation in Jimma city. The participants of this study stressed that increasing the number of ambulances was important in order to meet the demands of the community. To overcome EMS transportation scarcity, low-cost options such as retrofitted ambulances have been recommended in resource-limited areas, and to be complemented with currently available commercial and private transportations [2, 26, 29]. Moreover, we also recommend increased availability of professional care provider training programs by incorporating these courses into Ethiopian higher education institutions, in order to address growing demands for emergency care as a whole. Misuse of resources by ambulance providers leads to decreased utilization and mistrust of the system as a whole [6]. In the present study ambulance misuse was commonly felt to be a barrier to access in Jimma; the participants felt this was an impediment to trust in prehospital care and called for legal enforcement. At this time misuse of an ambulance is a prohibited practice under international humanitarian law and administrative actions [30], as well as existing local regulations [31, 32]. Therefore, legal enforcement is needed to halt abuse of this scarce resource. Overall, we stress that lack of proper EMS system means a higher number of deaths which could have been prevented easily.

\section{Limitations}

The participant interviews were conducted in Afan Oromo and Amharic, then translated and transcribed into English. Therefore, there was potential for mistranslation as many words in Afan Oromo are not 
directly translatable to English. Furthermore, there are likely opinions that were not represented in this study even though efforts were made to ensure diversity through involving participants from different parts of the community and relevant settings.

\section{Conclusion}

We identified that awareness in the community and accessibility of the service would have indispensible role in seeking and accessing prehospital emergency care in Jimma city. Community members face substantial barriers to care due to combination of limited public understanding of the role of prehospital care, lack of a single organized EMS system, lack of a toll-free emergency number, limited number of ambulances, lack of trained ambulance crews, poor road infrastructure and public mistrust of the existing system. Based on the experiences of other EMS systems in resource-limited settings of African nation, most solutions suggested by study participants seem feasible and applicable in this Ethiopian context, and should have to be considered in future interventions in prehospital care.

\section{Declarations}

\section{Ethics approval and consent to participate}

The study was approved by the Jimma University, Institute of Health IRB. Informed verbal consent was obtained from the participants, which was approved by the Jimma University, Institute of Health IRB.

\section{Consent for publication}

Not applicable

\section{Availability of data and materials}

The datasets used and/or analyzed during the current study are available from the corresponding author on reasonable request.

\section{Competing interests}

The authors declare that they have no competing interests.

\section{Funding}

This work was supported by Jimma University; the sponsor had no any involvement in study design; in the collection, analysis and interpretation of data, and in the writing of the report; and in the preparation 


\section{Authors' contributions}

SI, SS, NM, MA, AA and SW designed the study. SI, SS, AOA, TK, ES, TM, SC, GF, SW and GM acquired data. SI, AA and TK analyzed and interpreted data. Author SI, NM, ML, MA, HH, and ER prepared the manuscript. All authors reviewed the manuscript.

\section{Acknowledgement}

Authors acknowledge Jimma University for financial support to conduct this research.

\section{References}

[1]. WHO. Global, regional, and national life expectancy, all-cause and cause-specific mortality for 249 causes of death, 1980-2015: A systematic analysis for the global burden of disease study 2015. Geneva, Switzerland: WHO, 2015.

[2]. Razzak JA, Kellermann AL. Emergency medical care in developing countries: Is it worthwhile? Bulletin of the WHO. 2002;80:900-5.

[3]. Lopez A, Mathers C, Ezzati M, et al. Global and regional burden of disease and risk factors, 2001: Systematic analysis of population health data. Lancet. 2006;367(9524):10.

[4]. WHO. World health statistics 2010. Geneva, Switzerland: World Health Organization, 2010 September Report No.

[5]. Mock CN, Jurkovich G, nii-Amon-Kotei D, et al. Trauma mortality patterns in three nations at different economic levels: Implications for global trauma system development. . J Trauma. 1998;44:80412.

[6]. Stein C, Mould-Millman N-K, Vries SD, et al. Access to out-of-hospital emergency care in africa: Consensus conference recommendations. African Journal of Emergency Medicine. 2016;6:4.http://dx.doi.org/10.1016/j.afjem.2016.08.008.

[7]. Mould-Millman N-K, Naidoo R, Vries SD, et al. Afem out-of-hospital emergency care workgroup consensus paper: Advancing out-of-hospital emergency care in africa-advocacy and development. Afr $\mathrm{J}$ Emerg Med. 2014;4(2):5.

[8]. Mould-Millman N-K, Dixon JM, Sefa N, et al. The state of emergency medical services (ems) systems in africa. Prehosp Disaster Med. 2017;32(3):1-11.10.1017/S1049023X17000061. 
[9]. Reynolds TA, Calvello EJB, Broccoli MC, et al. Afem consensus conference 2013 summary: Emergency care in africa - where are we now? African Journal of Emergency Medicine 2014;4:15863.http://dx.doi.org/10.1016/j.afjem.2014.07.004.

[10]. Mould-Millman N-K, Rominski S, Bogus J, et al. Barriers to accessing emergency medical services in accra, ghana: Development of a survey instrument and initial application in ghana. Global Health Sci Pract. 2015;3(4):13.

[11]. Bosson N, Redlener M, Foltin G, et al. Barriers to utilization of pre-hospital emergency medical services among residents in libreville, gabon: A qualitative study. Afr J Emerg Med. 2013;3(4):5.

[12]. Broccoli MC, Calvello EJB, Skog AP, et al. Perceptions of emergency care in kenyan communities lacking access to formalised emergency medical systems: A qualitative study. BMJ Open. 2015;5.http://10.1136/bmjopen-2015-009208.

[13]. Mould-Millman N-K, editor Prehospital emergency care in sub-saharan africa: Innovations and challenges. African Federation of Emergency Medicine 2014.

[14]. Kironji AG, Hodkinson P, Ramirez SSd, et al. Identifying barriers for out of hospital emergency care in low and low-middle income countries: A systematic review. BMC Health Services Research. 2018;18(291 ):20.https://doi.org/10.1186/s12913-018-3091-0.

[15]. Mould-Millman N-K, Sasser SM, Wallis LA. Prehospital research in sub-saharan africa: Establishing research tenets. Academic Emergency medicine 2013;20(12):1304-9.

[16]. Moresky RT, Razzak J, Reynolds T, et al. Advancing research on emergency care systems in lowincome and middleincome countries: Ensuring high-quality care delivery systems. BMJ Glob Health. 2019;4.http://10.1136/bmjgh-2018-001265.

[17]. Kobusingye OC, Hyder AA, Bishai D, et al. Emergency medical systems in low- and middle-income countries: Recommendations for action. Bulletin of the World Health Organization 2005;83(8):626-31.

[18]. Menbeu Sultan, Yonas Abebe, Y. T, et al. Trends and barriers of emergency medical service use in addis ababa; ethiopia. BMC Emergency Medicine. 2019;19.10.1186/s12873-019-0242-5.

[19]. Sasser SM, Varghese M, Kellerman A, et al. Prehospital trauma care system. Geneva: 2005.

[20]. Mould-Millman N-K, Vries Sd, Stein C, et al. Developing emergency medical dispatch systems in africa - recommendations of the african federation for emergency medicine/international academies of emergency dispatch working African Journal of Emergency Medicine. 2015;5(3):6.https://doi.org/10.1016/j.afjem.2015.06.005.

[21]. Anon. Addis ababa city fire and emergency prevention and rescue agency 2016 [cited 2016 january 29]. Available from: http://www.addisababa.gov.et/fes 
[22]. Anon. Tebita ambulance and prehospital care service 2016 [cited 2016 February, 02]. Available from: http://www.tebitaambulance.com/\#home.

[23]. Waseem $H$, Naseer R, Razzak JA. Establishing a successful pre-hospital emergency service in a developing country: Experience from rescue 1122 service in pakistan. Emerg Med J. 2011;28: 513 $-15.10 .1136 / \mathrm{emj} .2010 .096271$.

[24]. Zimmerman J, Bertermann K, Bollinger $P$, et al. Prehospital system development in jaffna, sri lanka. Prehospital Disaster Medicine 2013;28(05):7.

[25]. Hsia RY, Thind A, Zakariah A, et al. Prehospital and emergency care: Updates from the disease control priorities, version 3 World J Surg. 2015.10.1007/s00268-015-2997-5.

[26]. Kobusingye OC. Emergency medical services - disease control priorities in developing countries NCBI bookshelf2012. Available from: http://www.ncbi.nlm.nih.gov/books/NBK11744/.

[27]. Wachira B, Martin IBK. The state of emergency care in the republic of kenya. Afr J Emerg Med. 2011;1:160-5.10.1016/j.afjem.2011.10.008.

[28]. Mould-Millman N-K, Rominski S, Oteng R. Ambulance or taxi? High acuity prehospital transports in the ashanti region of ghana. African Journal of Emergency Medicine. 2014;4:5.

[29]. Calvello E, Reynolds T, Hirshon JM, et al. Emergency care in sub-saharan africa: Results of a consensus conference. African Journal of Emergency Medicine 2013;3:7.http://dx.doi.org/10.1016/j.afjem.2013.01.001.

[30]. Anon. Ambulance and pre-hospital services in risk situations [Report]. In press 2013.

[31]. Legislation for ambulance service administration and utilization oromiya regional state, 148/2004 (2012).

[32]. ESA E. Ethiopian standard. General hospital - requirements. Ambulance service Addis Ababa, Ethiopia: Ethiopian Food, Medicine and Healthcare Administration and Control Authority; 2012. p. 3. 